\title{
BOUNDARY GROWTH OF SOBOLEV FUNCTIONS FOR DOUBLE PHASE FUNCTIONALS
}

\author{
Yoshihiro Mizuta and Tetsu Shimomura \\ 4-13-11 Hachi-Hom-Matsu-Minami \\ Higashi-Hiroshima 739-0144, Japan; yomizuta@hiroshima-u.ac.jp \\ Hiroshima University, Graduate School of Education, Department of Mathematics \\ Higashi-Hiroshima 739-8524, Japan; tshimo@hiroshima-u.ac.jp
}

\begin{abstract}
We study growth properties of spherical means of Sobolev functions for the double phase functional $\Phi_{p, q}(x, t)=t^{p}+(b(x) t)^{q}$ in the unit ball $\mathbf{B}$ of $\mathbf{R}^{n}$, where $1<p<q<\infty$ and $b(\cdot)$ is a non-negative bounded function on $\mathbf{B}$ which is Hölder continuous of order $\theta \in(0,1]$.
\end{abstract}

\section{Introduction}

Let $B(x, r)$ denote the open ball centered at $x \in \mathbf{R}^{n}(n \geq 2)$ of radius $r$, whose boundary is written as $S(x, r)$. For simplicity, the unit ball $B(0,1)$ and its boundary are written as $\mathbf{B}$ and $\mathbf{S}$, respectively. For a measurable function $f$ on the sphere $S(0, r)$, the $L^{\omega}$ mean for $1 \leq \omega<\infty$ is defined by

$$
S_{\omega}(f, r)=\left(\frac{1}{\sigma_{n} r^{n-1}} \int_{S(0, r)}|f(x)|^{\omega} d S(x)\right)^{1 / \omega},
$$

where $\sigma_{n}$ denotes the surface area of $S(0,1)$.

Gardiner [6, Theorem 2] proved that when $(n-3) /(n-1)<1 / \omega \leq(n-2) /(n-1)$ and $\omega \geq 1$,

$$
\liminf _{r \rightarrow 1}(1-r)^{n-1-(n-1) / \omega} S_{\omega}(G \mu, r)=0
$$

for a Green potential $G \mu$ on the unit ball B. Moreover, if $u$ is a $p$-precise function on B satisfying

$$
\int_{\mathbf{B}}|\nabla u(x)|^{p} d x<\infty
$$

then it is shown in [15] that

$$
\liminf _{r \rightarrow 1}(1-r)^{(n-p) / p-(n-1) / \omega} S_{\omega}(u, r)=0
$$

when $1<p<\omega<\infty$ and $0<(n-p) / p-(n-1) / \omega<1 / p$. See also Stoll [18].

Regarding regularity theory of differential equations, Baroni, Colombo and Mingione $[1,2,4,5]$ studied a double phase functional:

$$
\Phi(x, t)=\Phi_{p, q}(x, t)=t^{p}+(b(x) t)^{q},
$$

where $1<p<q<\infty$ and $b(\cdot)$ is a non-negative bounded function on $\mathbf{B}$ which is Hölder continuous of order $\theta \in(0,1]$. Harjulehto, Hästö and Karppinen [7] studied local higher integrability of the gradient of a quasiminimizer of the double phase functional. We refer to [4] for the minimization problem and [3] for the eigenvalue

https://doi.org/10.5186/aasfm.2020.4510

2010 Mathematics Subject Classification: Primary 31B25, 31B15.

Key words: Sobolev functions, spherical mean, double phase functional. 
problem for the double phase functional. See also [8] for the boundedness of maximal operators.

In this paper we study the growth properties for spherical means of $p$-precise functions $u$ in $\mathbf{B}$ satisfying

$$
\int_{\mathbf{B}} \Phi(x,|\nabla u(x)|) d x<\infty
$$

where $\nabla$ denotes the gradient. Our aim is to extend [15, Theorem 1] to the double phase functional $\Phi(x, t)$.

Theorem 1.1. Let $1<p<q<n, 1 / p-1 / q=\theta / n, \omega>q$ and

$$
0<\eta=\frac{n-(1+\theta) p}{p}-\frac{n-1}{\omega}=\frac{n-q}{q}-\frac{n-1}{\omega}<\frac{1}{q} .
$$

If $u$ is a $p$-precise function on $\mathbf{B}$ in the sense of Ziemer [20] satisfying (1.1), then

$$
\liminf _{t \rightarrow 1}(1-t)^{\eta} S_{\omega}(b u, t)=0 .
$$

The sharpness of the exponent $\eta$ in Theorem 1.1 will be discussed in the last section (Remark 7.2). We shall show that the lower limit can not be replaced by the upper limit (Remark 7.1). For the hyperplane case, we refer to [11, Theorem 2.1] in case $p=q$.

Example 1.2. Let $x_{0} \in \partial \mathbf{B}$ and $0<\theta \leq 1$. Examples of $b(x)$ are $\left|x-x_{0}\right|^{\theta}$ and $(1-|x|)^{\theta}$.

Next we are concerned with the case $\eta=0$.

Theorem 1.3. Let $1<p<q<n, 1 / p-1 / q=\theta / n, \omega>q$ and

$$
\frac{n-(1+\theta) p}{p}-\frac{n-1}{\omega}=\frac{n-q}{q}-\frac{n-1}{\omega}=0 .
$$

There exists a constant $C>0$ such that

$$
S_{\omega}(b u, t) \leq C
$$

for all p-precise function $u$ on $\mathbf{B}$ satisfying (1.1) and all $t>0$.

For the case $p=q$ in Theorem 1.3, see [15, Remark 3] and [11, Theorem 2.2]. We also refer to Yamashita [19] for harmonic functions $u$ on $\mathbf{B}$ satisfying (1.1) with $p=q=2$.

If $u$ is a $p$-precise function on $\mathbf{B}$, then the radial limit

$$
u(\xi)=\lim _{r \rightarrow 1} u(r \xi)
$$

exists for almost every $\xi \in \partial \mathbf{B}$. For this, we refer the reader to for example [12, Theorem 2.4, Chapter 8] in the half plane case; it suffices to see that $\int_{2^{-1}}^{1}\left|\frac{d}{d r} u(r \xi)\right| d r<\infty$ for almost every $\xi \in \partial \mathbf{B}$. Set

$$
U(r \xi)=u(r \xi)-u(\xi) \text { for } \xi \in \partial \mathbf{B} \text { and } 0<r<1 .
$$

Theorem 1.4. Let $1<p<q<n, 1 / p-1 / q=\theta / n, \omega>q$ and

$$
-1<\eta=\frac{n-(1+\theta) p}{p}-\frac{n-1}{\omega}=\frac{n-q}{q}-\frac{n-1}{\omega}<0 .
$$

If $u$ is a $p$-precise function on $\mathbf{B}$ satisfying (1.1), then

$$
\lim _{t \rightarrow 1}(1-t)^{\eta} S_{\omega}(b U, t)=0 .
$$


We refer to [11, Theorem 3.1] for the hyperplane case and $p=q$. See [16] for monotone Sobolev functions as a generalization of harmonic functions.

Throughout this paper, let $C$ denote various constants independent of the variables in question.

\section{2. $p$-precise function}

If $0<\alpha<n, 1<p<\infty, G$ is an open set in $\mathbf{R}^{n}$ and $E \subset G$, then the relative $(\alpha, p)$-capacity is defined by

$$
C_{\alpha, p}^{(n)}(E ; G)=\inf \int_{G} f(y)^{p} d y
$$

where the infimum is taken over all nonnegative measurable functions $f$ on $G$ such that

$$
\int_{G}|x-y|^{\alpha-n} f(y) d y \geq 1 \quad \text { for every } x \in E
$$

see [9] and [12] for the basic properties of $(\alpha, p)$-capacity. We say that a set $E$ has $(\alpha, p)$-capacity zero if $C_{\alpha, p}^{(n)}(E \cap G ; G)=0$ for every bounded open set $G \subset \mathbf{R}^{n}$.

Following Ziemer [20], we say that a locally integrable function $u$ is $p$-precise in $\mathrm{B}$ if

(i) $\int_{\mathbf{B}}|\nabla u(x)|^{p} d x<\infty$, where $\nabla$ denotes the gradient;

(ii) $u$ is quasicontinuous in $\mathbf{B}$, in the sense that for every $\varepsilon>0$, there exists an open set $G$ such that $C_{1, p}^{(n)}(G ; \mathbf{B})<\varepsilon$ and $u$ is continuous as a function on B \G.

We note that if $u$ is $p$-precise in $\mathbf{B}$, then $u$ is partially differentiable almost everywhere on $\mathbf{B}$ and its spherical means over $S(x, r)$ are well defined whenever $S(x, r) \subset \mathbf{B}$, since a set of $(1, p)$-capacity zero has Hausdorff dimension at most $n-p$.

\section{Lemmas}

Let us begin with the following lemma.

Lemma 3.1. (cf. [13, Lemma 2.1], [14, Lemma 2.2]) Let $0<a<1$ and $c_{1}$ be positive constants. Then there exists a constant $C>0$ such that

$$
\int_{S(0,1)}|t \sigma-y|^{a-n} d S(\sigma) \leq C|t-| y||^{a-1},
$$

whenever $y \in \mathbf{R}^{n}$ and $1 / 2<t<\min \left\{1, c_{1}|y|\right\}$.

In fact, this holds for $y \in \mathbf{B}$ by [13, Lemma 2.1]. With a slight modification of the proof of [13, Lemma 2.1], this holds for all $y \in \mathbf{R}^{n}$. by

For $0<\alpha<n$ and a nonnegative measurable function $f$ on $\mathbf{R}^{n}$, we define $R_{\alpha} f$

$$
R_{\alpha} f(x)=\int_{\mathbf{R}^{n}}|x-y|^{\alpha-n} f(y) d y .
$$

Lemma 3.2. Let $1<p \leq \omega<\infty$ and

$$
\eta=\frac{n-\alpha p}{p}-\frac{n-1}{\omega}>0
$$


Let $f$ be a nonnegative measurable function on $B(0,2) \backslash \mathbf{B}$ satisfying

$$
\int_{B(0,2) \backslash \mathbf{B}}|f(y)|^{p} d y<\infty .
$$

Then

$$
\lim _{t \rightarrow 1}(1-t)^{\eta} S_{\omega}\left(R_{\alpha} f, t\right)=0 .
$$

Proof. Let $f$ be a nonnegative measurable function in $L^{p}(B(0,2) \backslash \mathbf{B})$. For $\varepsilon>0$ and $x \in \mathbf{B}$ we have by Hölder's inequality

$$
\begin{aligned}
R_{\alpha} f(x) & \leq\left(\int_{B(0,2) \backslash \mathbf{B}}|x-y|^{-\varepsilon p^{\prime}-n} d y\right)^{1 / p^{\prime}}\left(\int_{B(0,2) \backslash \mathbf{B}}|x-y|^{\alpha p+\varepsilon p-n} f(y)^{p} d y\right)^{1 / p} \\
& \leq C(1-|x|)^{-\varepsilon}\left(\int_{B(0,2) \backslash \mathbf{B}}|x-y|^{\alpha p+\varepsilon p-n} f(y)^{p} d y\right)^{1 / p} .
\end{aligned}
$$

Hence we see from Minkowski's inequality and Lemma 3.1 that

$$
\begin{aligned}
(1-t)^{\eta} S_{\omega}\left(R_{\alpha} f, t\right) & \leq C(1-t)^{\eta-\varepsilon}\left(\int_{B(0,2) \backslash \mathbf{B}}\left(S_{\omega}\left(|\cdot-y|^{(\alpha p+\varepsilon p-n) / p}, t\right)\right)^{p} f(y)^{p} d y\right)^{1 / p} \\
& \leq C(1-t)^{\eta-\varepsilon+(\alpha p+\varepsilon p-n) / p+(n-1) / \omega}\left(\int_{B(0,2) \backslash \mathbf{B}} f(y)^{p} d y\right)^{1 / p} \\
& =C\left(\int_{B(0,2) \backslash \mathbf{B}} f(y)^{p} d y\right)^{1 / p}
\end{aligned}
$$

when $0<\varepsilon<\eta$, so that

$$
\limsup _{t \rightarrow 1}(1-t)^{\eta} S_{\omega}\left(R_{\alpha} f, t\right) \leq C\left(\int_{B(0,2) \backslash \mathbf{B}} f(y)^{p} d y\right)^{1 / p} .
$$

For $1<r<2$, write

$$
\begin{aligned}
R_{\alpha} f(x) & =\int_{B(0,2) \backslash B(0, r)}|x-y|^{\alpha-n} f(y) d y+\int_{B(0, r) \backslash \mathbf{B}}|x-y|^{\alpha-n} f(y) d y \\
& =I_{1}(x)+I_{2}(x) .
\end{aligned}
$$

Now, since $I_{1}$ is bounded in $\mathbf{B}$, we see that

$$
\begin{aligned}
\limsup _{t \rightarrow 1}(1-t)^{\eta} S_{\omega}\left(R_{\alpha} f, t\right) & =\limsup _{t \rightarrow 1}(1-t)^{\eta} S_{\omega}\left(I_{2}, t\right) \\
& \leq C\left(\int_{B(0, r) \backslash \mathbf{B}} f(y)^{p} d y\right)^{1 / p}
\end{aligned}
$$

for all $1<r<2$, which gives the result.

We introduce Sobolev's integral representation. If $1<q<\infty$ and $v \in C_{0}^{\infty}\left(\mathbf{R}^{n}\right)$, then

$$
v(x)=c \sum_{i=1}^{n} \int \frac{x_{i}-y_{i}}{|x-y|^{n}} \frac{\partial v}{\partial y_{i}} d y
$$

holds for all $x \in \mathbf{R}^{n}$, where $c=1 / \sigma_{n}$ [15, Lemma 2]. 
Lemma 3.3. [15, Corollary 3] Let $u$ be a $p$-precise function on $\mathbf{B}$. Then there is a $p$-precise function $\bar{u}$ on $\mathbf{R}^{n}$ such that

$$
u(x)=c \sum_{i=1}^{n} \int \frac{x_{i}-y_{i}}{|x-y|^{n}} \frac{\partial \bar{u}}{\partial y_{i}} d y
$$

holds on $\mathbf{B}$ except for a set of $C_{1, p}^{(n)}$ capacity zero.

Here $\bar{u}$ is a $p$-precise extension of $u$ to $\mathbf{R}^{n}$ with compact support by Stein [17, Chapter 5], or we may consider the inversion to define $\bar{u}(x)=u\left(x /|x|^{2}\right)$ for $|x|>1$.

Lemma 3.4. [10, Lemma 5.2], [13, Lemma 2.7] Let $0<\alpha_{1}, \alpha_{2}<1$ and $\mu_{1}, \mu_{2}$ be nonnegative measures on $\mathbf{R}^{+}=(0, \infty)$ such that $\mu_{1}\left(\mathbf{R}^{+}\right)+\mu_{2}\left(\mathbf{R}^{+}\right)<\infty$. Set

$$
G(x)=x^{\alpha_{1}} \int_{A(x)}|x-y|^{-\alpha_{1}} d \mu_{1}(y)+x^{\alpha_{2}} \int_{A(x)}|x-y|^{-\alpha_{2}} d \mu_{2}(y),
$$

where $A(x)=\{y: x / 2<y<2 x\}$. Then

$$
\liminf _{x \rightarrow 0+} G(x)=0 .
$$

Proof. For a positive integer $j$ we have by Fubini's theorem

$$
\begin{aligned}
\int_{2^{-j}}^{2^{-j+1}} G(x) \frac{d x}{x} \leq & \int_{2^{-j}}^{2^{-j+1}}\left(2^{(-j+1) \alpha_{1}} \int_{2^{-j-1}}^{2^{-j+2}}|x-y|^{-\alpha_{1}} d \mu_{1}(y)\right) \frac{d x}{x} \\
& +\int_{2^{-j}}^{2^{-j+1}}\left(2^{(-j+1) \alpha_{2}} \int_{2^{-j-1}}^{2^{-j+2}}|x-y|^{-\alpha_{2}} d \mu_{2}(y)\right) \frac{d x}{x} \\
= & 2^{(-j+1) \alpha_{1}} \int_{2^{-j-1}}^{2^{-j+2}}\left(\int_{2^{-j}}^{2^{-j+1}}|x-y|^{-\alpha_{1}} \frac{d x}{x}\right) d \mu_{1}(y) \\
& +2^{(-j+1) \alpha_{2}} \int_{2^{-j-1}}^{2^{-j+2}}\left(\int_{2^{-j}}^{2^{-j+1}}|x-y|^{-\alpha_{2}} \frac{d x}{x}\right) d \mu_{2}(y) \\
\leq & C 2^{(-j+1) \alpha_{1}} 2^{j \alpha_{1}} \int_{2^{-j-1}}^{2^{-j+2}} d \mu_{1}(y)+C 2^{(-j+1) \alpha_{1}} 2^{j \alpha_{2}} \int_{2^{-j-1}}^{2^{-j+2}} d \mu_{2}(y) \\
\leq & C\left(\int_{2^{-j-1}}^{2^{-j+2}} d \mu_{1}(y)+\int_{2^{-j-1}}^{2^{-j+2}} d \mu_{2}(y)\right)
\end{aligned}
$$

so that

$$
\lim _{j \rightarrow \infty} \inf _{2^{-j}<x<2^{-j+1}} G(x)=0
$$

which proves the result.

By change of variables $x=1-t$ and $y=1-s$, we obtain the following result.

Corollary 3.5. [10, Corollary 5.1] Let $0<\alpha_{1}, \alpha_{2}<1$ and $\mu_{1}, \mu_{2}$ be nonnegative measures on $I=(0,1)$ such that $\mu_{1}(I)+\mu_{2}(I)<\infty$. Then

$$
\liminf _{t \rightarrow 1-}\left((1-t)^{\alpha_{1}} \int_{A(0, t)}|t-s|^{-\alpha_{1}} d \mu_{1}(s)+(1-t)^{\alpha_{2}} \int_{A(0, t)}|t-s|^{-\alpha_{2}} d \mu_{2}(s)\right)=0
$$

where $A(0, t)=\{s: t-(1-t) / 2<s<t+(1-t) / 2\}$. 
Write

$$
\begin{aligned}
R_{1} f(x)= & \int_{\mathbf{R}^{n}}|x-y|^{1-n} f(y) d y \\
= & \int_{B(x,(1-|x|) / 2)}|x-y|^{1-n} f(y) d y \\
& +\int_{\left\{y \in \mathbf{R}^{n} \backslash B(x,(1-|x|) / 2): 1-|y| \leq 1-|x|\right\}}|x-y|^{1-n} f(y) d y \\
& +\int_{\left\{y \in \mathbf{R}^{n} \backslash B(x,(1-|x|) / 2): 1-|y|>1-|x|\right\}}|x-y|^{1-n} f(y) d y \\
= & I_{1}(x)+I_{2}(x)+I_{3}(x) .
\end{aligned}
$$

Lemma 3.6. Let $1<p<q<\omega<\infty, 1 / p-1 / q=\theta / n$ and

$$
\eta=\frac{n-(1+\theta) p}{p}-\frac{n-1}{\omega}=\frac{n-q}{q}-\frac{n-1}{\omega}>0 .
$$

Let $f$ be a nonnegative measurable function on $\mathbf{R}^{n}$ satisfying

$$
\int_{\mathbf{B}} \Phi(x, f(x)) d x<\infty .
$$

Then

$$
\liminf _{t \rightarrow 1}(1-t)^{\eta} S_{\omega}\left(b I_{1}, t\right)=0
$$

Proof. Let $f$ be a nonnegative measurable function on $\mathbf{R}^{n}$ satisfying (3.1). We have

$$
\begin{aligned}
& b(x) I_{1}(x) \\
& =\int_{B(x,(1-|x|) / 2)}|x-y|^{1-n}(b(x)-b(y)) f(y) d y+\int_{B(x,(1-|x|) / 2)}|x-y|^{1-n} b(y) f(y) d y \\
& \leq C \int_{B(x,(1-|x|) / 2)}|x-y|^{1+\theta-n} f(y) d y+\int_{B(x,(1-|x|) / 2)}|x-y|^{1-n} b(y) f(y) d y \\
& =C I_{11}(x)+I_{12}(x) .
\end{aligned}
$$

Take $\delta_{1}, \delta_{2} \in(0,1)$ such that

$$
0<\eta p<\beta_{1}=(n-1-\theta) \delta_{1}-p(n-1) / \omega<1
$$

and

$$
0<\eta q<\beta_{2}=(n-1) \delta_{2}-q(n-1) / \omega<1 .
$$

Then we have by Hölder's inequality

$$
\begin{aligned}
I_{11}(x) \leq & \left(\int_{B(x,(1-|x|) / 2)}|x-y|^{(1+\theta-n)\left(1-\delta_{1} / p\right) p^{\prime}} d y\right)^{1 / p^{\prime}} \\
& \cdot\left(\int_{B(x,(1-|x|) / 2)}|x-y|^{(1+\theta-n) \delta_{1}} f(y)^{p} d y\right)^{1 / p} \\
\leq & C(1-|x|)^{(1+\theta-n)\left(1-\delta_{1} / p\right)+n / p^{\prime}}\left(\int_{B(x,(1-|x|) / 2)}|x-y|^{(1+\theta-n) \delta_{1}} f(y)^{p} d y\right)^{1 / p} \\
\leq & C(1-t)^{\beta_{1} / p-\eta}\left(\int_{A(0, t)}|x-y|^{-\beta_{1}-p(n-1) / \omega} f(y)^{p} d y\right)^{1 / p}
\end{aligned}
$$


since $(1+\theta-n)\left(1-\delta_{1} / p\right)+n / p^{\prime}=\beta_{1} / p-\eta>0$ and $B(x,(1-|x|) / 2) \subset\{y \in$ B: $t-(1-t) / 2<|y|<t+(1-t) / 2\}=A(0, t)$ when $t=|x|$. Hence we see from Minkowski's inequality and Lemma 3.1 that

$$
\begin{aligned}
(1-t)^{\eta} S_{\omega}\left(I_{11}, t\right) & \leq C(1-t)^{\beta_{1} / p}\left(\int_{A(0, t)}\left(S_{\omega}\left(|\cdot-y|^{\left(-\beta_{1}-p(n-1) / \omega\right) / p}, t\right)\right)^{p} f(y)^{p} d y\right)^{1 / p} \\
& \leq C\left(\left.(1-t)^{\beta_{1}} \int_{A(0, t)}|t-| y\right|^{-\beta_{1}} f(y)^{p} d y\right)^{1 / p} .
\end{aligned}
$$

Thus it follows from Corollary 3.5 and (3.1) that

$$
\begin{aligned}
& \liminf _{t \rightarrow 1}(1-t)^{\eta} S_{\omega}\left(b I_{1}, t\right) \\
& \leq C \liminf _{t \rightarrow 1}\left\{(1-t)^{\eta} S_{\omega}\left(I_{11}, t\right)+(1-t)^{\eta} S_{\omega}\left(I_{12}, t\right)\right\}=0,
\end{aligned}
$$

as required.

\section{Proof of Theorem 1.1}

Let $u$ be a $p$-precise function on $\mathbf{B}$ satisfying (1.1). By Lemma 3.3, we have

$$
|u(x)| \leq \frac{1}{\sigma_{n}} \int_{\mathbf{R}^{n}}|x-y|^{1-n}|\nabla \bar{u}(y)| d y=\frac{1}{\sigma_{n}} R_{1} f(x)
$$

on B except for a set of capacity zero, where $f(y)=|\nabla \bar{u}(y)|$. Here we may assume that the extension $\bar{u}$ vanishes outside $B(0,2)$. Write

$$
\begin{aligned}
b(x) I_{2}(x)= & \int_{\left\{y \in \mathbf{R}^{n} \backslash B(x,(1-|x|) / 2): 1-|y| \leq 1-|x|\right\}}|x-y|^{1-n}(b(x)-b(y)) f(y) d y \\
& +\int_{\left\{y \in \mathbf{R}^{n} \backslash B(x,(1-|x|) / 2): 1-|y| \leq 1-|x|\right\}}|x-y|^{1-n} b(y) f(y) d y \\
\leq & C \int_{\left\{y \in \mathbf{R}^{n} \backslash B(x,(1-|x|) / 2): 1-|y| \leq 1-|x|\right\}}|x-y|^{1+\theta-n} f(y) d y \\
& +\int_{\left\{y \in \mathbf{R}^{n} \backslash B(x,(1-|x|) / 2): 1-|y| \leq 1-|x|\right\}}|x-y|^{1-n} b(y) f(y) d y \\
= & C I_{21}(x)+I_{22}(x) .
\end{aligned}
$$

Further, set

$$
\begin{aligned}
& I_{211}(x)=\int_{\{y \in \mathbf{B} \backslash B(x,(1-|x|) / 2): 1-|y| \leq 1-|x|\}}|x-y|^{1+\theta-n} f(y) d y, \\
& I_{212}(x)=\int_{B(0,2) \backslash \mathbf{B}}|x-y|^{1+\theta-n} f(y) d y .
\end{aligned}
$$

Note here that

$$
(1+\theta) p-n+\frac{n-1}{\omega / p}=-p \eta<0 .
$$

Since $f \in L^{p}\left(\mathbf{R}^{n}\right)$, we apply [13, Lemma $\left.2.8(1)\right]$ to obtain

$$
\lim _{t \rightarrow 1}(1-t)^{\eta} S_{\omega}\left(I_{211}, t\right)=0 \text {. }
$$

In view of Lemma 3.2 with $\alpha=1+\theta$, we also obtain

$$
\lim _{t \rightarrow 1}(1-t)^{\eta} S_{\omega}\left(I_{212}, t\right)=0
$$


so that

$$
\lim _{t \rightarrow 1}(1-t)^{\eta} S_{\omega}\left(I_{21}, t\right)=0 .
$$

Similarly, noting that $1-n+(n-1) /(\omega / q)=-q \eta<0$ and $b f \in L^{q}\left(\mathbf{R}^{n}\right)$, we find

$$
\lim _{t \rightarrow 1}(1-t)^{\eta} S_{\omega}\left(I_{22}, t\right)=0 .
$$

Hence,

$$
\lim _{t \rightarrow 1}(1-t)^{\eta} S_{\omega}\left(b I_{2}, t\right)=0 .
$$

For the estimate of $I_{3}$ we have

$$
\begin{aligned}
b(x) I_{3}(x) \leq & C \int_{\left\{y \in \mathbf{R}^{n} \backslash B(x,(1-|x|) / 2): 1-|y|>1-|x|\right\}}|x-y|^{1+\theta-n} f(y) d y \\
& +\int_{\left\{y \in \mathbf{R}^{n} \backslash B(x,(1-|x|) / 2): 1-|y|>1-|x|\right\}}|x-y|^{1-n} b(y) f(y) d y \\
= & C I_{31}(x)+I_{32}(x) .
\end{aligned}
$$

Since $(1+\theta) p-n+p(n-1) / \omega<0$ and $f \in L^{p}\left(\mathbf{R}^{n}\right)$, in view of [13, Lemma 2.9 (1)], we obtain

$$
\lim _{t \rightarrow 1}(1-t)^{\eta} S_{\omega}\left(I_{31}, t\right)=0
$$

and since $1-n+q(n-1) / \omega<0$ and $b f \in L^{q}\left(\mathbf{R}^{n}\right)$,

$$
\lim _{t \rightarrow 1}(1-t)^{\eta} S_{\omega}\left(I_{32}, t\right)=0,
$$

so that

$$
\lim _{t \rightarrow 1}(1-t)^{\eta} S_{\omega}\left(b I_{3}, t\right)=0 .
$$

Therefore, Lemma 3.6 gives

$$
\liminf _{t \rightarrow 1}(1-t)^{\eta} S_{\omega}(b u, t)=0 .
$$

This completes the proof of the theorem.

\section{Proof of Theorem 1.3}

Noting (4.1), we write

$$
\begin{aligned}
b(x) R_{1} f(x) & =\int_{\mathbf{R}^{n}}|x-y|^{1-n}(b(x)-b(y)) f(y) d y+\int_{\mathbf{R}^{n}}|x-y|^{1-n} b(y) f(y) d y \\
& \leq C \int_{\mathbf{R}^{n}}|x-y|^{1+\theta-n} f(y) d y+\int_{\mathbf{R}^{n}}|x-y|^{1-n} b(y) f(y) d y \\
& =J_{1}(x)+J_{2}(x),
\end{aligned}
$$

where $f(y)=|\nabla \bar{u}(y)|$ as before. Here we may assume that the extension $\bar{u}$ vanishes outside $B(0,2)$. As in the proof of [11, Theorem 2.2] ([15, Remark 3]), we have by Hölder's inequality

$$
\begin{aligned}
\left|J_{1}(x)\right| & \leq C \int_{S(0,1)}\left(\int_{0}^{2}\left|x-s y^{*}\right|^{(1+\theta-n) p^{\prime}} s^{n-1} d t\right)^{1 / p^{\prime}}\left(\int_{0}^{2} f\left(s y^{*}\right)^{p} s^{n-1} d t\right)^{1 / p} d S\left(y^{*}\right) \\
& \leq C \int_{S(0,1)}\left|x^{*}-y^{*}\right|^{1+\theta-n+1 / p^{\prime}}\left(\int_{0}^{2} f\left(s y^{*}\right)^{p} s^{n-1} d t\right)^{1 / p} d S\left(y^{*}\right)
\end{aligned}
$$


and

$$
\left|J_{2}(x)\right| \leq C \int_{S(0,1)}\left|x^{*}-y^{*}\right|^{1-n+1 / p^{\prime}}\left(\int_{0}^{2}\left(b\left(s y^{*}\right) f\left(s y^{*}\right)\right)^{p} s^{n-1} d t\right)^{1 / p} d S\left(y^{*}\right),
$$

where $x^{*}=x /|x|$ and $y^{*}=y /|y|$. Applying Sobolev's inequality, we obtain

$$
S_{\omega}\left(J_{1}, t\right) \leq C\left(\int_{\mathbf{R}^{n}} f(x)^{p} d x\right)^{1 / p}
$$

and

$$
S_{\omega}\left(J_{2}, t\right) \leq C\left(\int_{\mathbf{R}^{n}}(b(x) f(x))^{q} d x\right)^{1 / q}
$$

where

$$
\frac{n-(1+\theta) p}{p}-\frac{n-1}{\omega}=\frac{n-q}{q}-\frac{n-1}{\omega}=0 .
$$

Therefore, in view of (4.1), we find

$$
S_{\omega}(b u, t) \leq C,
$$

as required.

\section{Proof of Theorem 1.4}

Let $u$ be a $p$-precise function on $\mathbf{B}$ satisfying (1.1). In view of (1.2), we have

$$
U(x)=\frac{1}{\sigma_{n}} \sum_{i=1}^{n} \int_{\mathbf{R}^{n}} K_{i}(x, y) D_{i} u(y) d y
$$

where $K_{i}(x, y)=\left(x_{i}-y_{i}\right)|x-y|^{-n}-\left(\xi_{i}-y_{i}\right)|\xi-y|^{-n}$ with $\xi=x /|x|$ and $D_{i} u=$ $\left(\partial / \partial y_{i}\right) u$. Write

$$
\begin{aligned}
b(x) U(x)= & \frac{1}{\sigma_{n}} \sum_{i=1}^{n} \int_{\left\{y \in \mathbf{R}^{n}: 2(1-|x|)<1-|y|\right\}} K_{i}(x, y)(b(x)-b(y)) D_{i} u(y) d y \\
& +\frac{1}{\sigma_{n}} \sum_{i=1}^{n} \int_{\left\{y \in \mathbf{R}^{n}: 2(1-|x|)<1-|y|\right\}} K_{i}(x, y)\left(b(y) D_{i} u(y)\right) d y \\
& +\frac{1}{\sigma_{n}} \sum_{i=1}^{n} \int_{\left\{y \in \mathbf{R}^{n}:(1-|y|) / 2<1-|x|<2(1-|y|)\right\}} K_{i}(x, y)(b(x)-b(y)) D_{i} u(y) d y \\
& +\frac{1}{\sigma_{n}} \sum_{i=1}^{n} \int_{\left\{y \in \mathbf{R}^{n}:(1-|y|) / 2<1-|x|<2(1-|y|)\right\}} K_{i}(x, y)\left(b(y) D_{i} u(y)\right) d y \\
& +\frac{1}{\sigma_{n}} \sum_{i=1}^{n} \int_{\left\{y \in \mathbf{R}^{n}: 2(1-|y|)<1-|x|\right\}} K_{i}(x, y)(b(x)-b(y)) D_{i} u(y) d y \\
& +\frac{1}{\sigma_{n}} \sum_{i=1}^{n} \int_{\left\{y \in \mathbf{R}^{n}: 2(1-|y|)<1-|x|\right\}} K_{i}(x, y)\left(b(y) D_{i} u(y)\right) d y \\
= & I_{11}(x)+I_{12}(x)+I_{21}(x)+I_{22}(x)+I_{31}(x)+I_{32}(x) .
\end{aligned}
$$

First we treat $I_{11}$ and $I_{12}$. Since

$$
\left|K_{i}(x, y)\right| \leq C(1-|x|)|x-y|^{-n} \quad \text { when } 2(1-|x|)<1-|y|,
$$


we have

$$
\left|I_{11}\right| \leq C(1-|x|) \int_{\left\{y \in \mathbf{R}^{n}: 2(1-|x|)<1-|y|\right\}}|x-y|^{\theta-n}|\nabla u(y)| d y
$$

and

$$
\left|I_{12}\right| \leq C(1-|x|) \int_{\left\{y \in \mathbf{R}^{n}: 2(1-|x|)<1-|y|\right\}}|x-y|^{-n} b(y)|\nabla u(y)| d y .
$$

Therefore

$$
S_{\omega}\left(I_{11}, t\right) \leq C(1-t)\left(\int_{\left\{y \in \mathbf{R}^{n}: 2(1-t)<1-|y|\right\}}(1-|y|)^{(\theta p-n)+p(n-1) / \omega}|\nabla u(y)|^{p} d y\right)^{1 / p}
$$

and

$$
S_{\omega}\left(I_{12}, t\right) \leq C(1-t)\left(\int_{\left\{y \in \mathbf{R}^{n}: 2(1-t)<1-|y|\right\}}(1-|y|)^{-n+q(n-1) / \omega}(b(y)|\nabla u(y)|)^{q} d y\right)^{1 / q} .
$$

Hence it follows from Lebesgue's dominated convergence theorem that

$$
\begin{aligned}
& \limsup _{t \rightarrow 1}(1-t)^{\eta} S_{\omega}\left(I_{11}, t\right) \\
& \leq C \limsup _{t \rightarrow 1}\left(\int_{\left\{y \in \mathbf{R}^{n}: 2(1-t)<1-|y|\right\}}\left(\frac{1-t}{1-|y|}\right)^{(1+\eta) p}|\nabla u(y)|^{p} d y\right)^{1 / p}=0
\end{aligned}
$$

and

$$
\begin{aligned}
& \limsup _{t \rightarrow 1}(1-t)^{\eta} S_{\omega}\left(I_{12}, t\right) \\
& \leq C \limsup _{t \rightarrow 1}\left(\int_{\left\{y \in \mathbf{R}^{n}: 2(1-t)<1-|y|\right\}}\left(\frac{1-t}{1-|y|}\right)^{(1+\eta) q}(b(y)|\nabla u(y)|)^{q} d y\right)^{1 / q}=0 .
\end{aligned}
$$

To treat $I_{21}$ and $I_{22}$, noting that

$$
\left|K_{i}(x, y)\right| \leq C|x-y|^{1-n} \quad \text { when }(1-|y|) / 2<1-|x|<2(1-|y|),
$$

we have

$$
\left|I_{21}\right| \leq C \int_{\left\{y \in \mathbf{R}^{n}:(1-|y|) / 2<1-|x|<2(1-|y|)\right\}}|x-y|^{(1+\theta)-n}|\nabla u(y)| d y
$$

and

$$
\left|I_{22}\right| \leq C \int_{\left\{y \in \mathbf{R}^{n}:(1-|y|) / 2<1-|x|<2(1-|y|)\right\}}|x-y|^{1-n} b(y)|\nabla u(y)| d y .
$$

Therefore

$$
\begin{aligned}
& S_{\omega}\left(I_{21}, t\right) \leq C(1-|x|)^{((1+\theta) p-n) / p+(n-1) / \omega} \\
& \quad \cdot\left(\int_{\left\{y \in \mathbf{R}^{n}:(1-|y|) / 2<1-|x|<2(1-|y|)\right\}}|\nabla u(y)|^{p} d y\right)^{1 / p}
\end{aligned}
$$

and

$$
\begin{aligned}
& S_{\omega}\left(I_{22}, t\right) \leq C(1-|y|)^{(q-n) / q+(n-1) / \omega} \\
& \quad \cdot\left(\int_{\left\{y \in \mathbf{R}^{n}:(1-|y|) / 2<1-|x|<2(1-|y|)\right\}}(b(y)|\nabla u(y)|)^{q} d y\right)^{1 / q} .
\end{aligned}
$$


Hence

$$
\begin{aligned}
& \limsup _{t \rightarrow 1}(1-t)^{\eta} S_{\omega}\left(I_{21}, t\right) \\
& \leq C \limsup _{t \rightarrow 1}\left(\int_{\left\{y \in \mathbf{R}^{n}:(1-|y|) / 2<1-t<2(1-|y|)\right\}}|\nabla u(y)|^{p} d y\right)^{1 / p}=0
\end{aligned}
$$

and

$$
\begin{aligned}
& \limsup _{t \rightarrow 1}(1-t)^{\eta} S_{\omega}\left(I_{22}, t\right) \\
& \leq C \limsup _{t \rightarrow 1}\left(\int_{\left\{y \in \mathbf{R}^{n}:(1-|y|) / 2<1-t<2(1-|y|)\right\}}(b(y)|\nabla u(y)|)^{q} d y\right)^{1 / q}=0 .
\end{aligned}
$$

Finally we treat $I_{31}$ and $I_{32}$. Since

$$
\left|K_{i}(x, y)\right| \leq C|\xi-y|^{1-n} \quad \text { when } 2(1-|y|)<1-|x|,
$$

we have

$$
\left|I_{31}\right| \leq C \int_{\left\{y \in \mathbf{R}^{n}: 2(1-|y|)<1-|x|\right\}}|\xi-y|^{1+\theta-n}|\nabla u(y)| d y
$$

and

$$
\left|I_{32}\right| \leq C \int_{\left\{y \in \mathbf{R}^{n}: 2(1-|y|)<1-|x|\right\}}|\xi-y|^{1-n} b(y)|\nabla u(y)| d y
$$

Therefore

$$
S_{\omega}\left(I_{31}, t\right) \leq C\left(\int_{\left\{y \in \mathbf{R}^{n}: 2(1-|y|)<1-t\right\}}(1-|y|)^{((1+\theta) p-n)+p(n-1) / \omega}|\nabla u(y)|^{p} d y\right)^{1 / p}
$$

and

$$
S_{\omega}\left(I_{32}, t\right) \leq C\left(\int_{\left\{y \in \mathbf{R}^{n}: 2(1-|y|)<1-t\right\}}(1-|y|)^{-n+q+q(n-1) / \omega}(b(y)|\nabla u(y)|)^{q} d y\right)^{1 / q} .
$$

Hence, by Lebesgue's dominated convergence theorem, we have

$$
\begin{aligned}
& \limsup _{t \rightarrow 1}(1-t)^{\eta} S_{\omega}\left(I_{31}, t\right) \\
& \leq C \limsup _{t \rightarrow 1}\left(\int_{\left\{y \in \mathbf{R}^{n}: 2(1-|y|)<1-t\right\}}\left(\frac{1-t}{1-|y|}\right)^{\eta p}|\nabla u(y)|^{p} d y\right)^{1 / p}=0
\end{aligned}
$$

and

$$
\begin{aligned}
& \limsup _{t \rightarrow 1}(1-t)^{\eta} S_{\omega}\left(I_{32}, t\right) \\
& \leq C \limsup _{t \rightarrow 1}\left(\int_{\left\{y \in \mathbf{R}^{n}: 2(1-|y|)<1-t\right\}}\left(\frac{1-t}{1-|y|}\right)^{\eta q}(b(y)|\nabla u(y)|)^{q} d y\right)^{1 / q}=0 .
\end{aligned}
$$

Now we establish

$$
\lim _{t \rightarrow 1}(1-t)^{\eta} S_{\omega}(b U, t)=0
$$

as required. 


\section{Remarks}

Remark 7.1. We show that the lower limit in Theorem 1.1 can not be replaced by the upper limit. To show this, for $x_{0} \in \partial \mathbf{B}, a>0$ and $0<\theta \leq 1$, letting $x_{j}=\left(1-2^{-j}\right) x_{0}$, we consider

$$
u(x)=\sum_{j=1}^{\infty} u_{j}(x)=\sum_{j=1}^{\infty} 2^{-j}\left|x-x_{j}\right|^{-a} \varphi\left(2^{j+3}\left|x-x_{j}\right|\right)
$$

and

$$
b(x)=\sum_{j=1}^{\infty} b_{j}(x)=\sum_{j=1}^{\infty} 2^{-j}\left|x-x_{j}\right|^{\theta} \varphi\left(2^{j+3}\left|x-x_{j}\right|\right),
$$

where

$$
\varphi(t)= \begin{cases}1 & (0<t<1) \\ 2-t & (1 \leq t<2) \\ 0 & (t \geq 2)\end{cases}
$$

Then we have for $x, y \in B\left(x_{j}, 2^{-j-2}\right)$

$$
\begin{aligned}
\left|b_{j}(x)-b_{j}(y)\right| \leq & 2^{-j}|| x-\left.x_{j}\right|^{\theta}-\left|y-x_{j}\right|^{\theta} \mid \varphi\left(2^{j+3}\left|x-x_{j}\right|\right) \\
& +2^{-j}\left|y-x_{j}\right|^{\theta}\left|\varphi\left(2^{j+3}\left|x-x_{j}\right|\right)-\varphi\left(2^{j+3}\left|y-x_{j}\right|\right)\right| \\
\leq & 2^{-j}|x-y|^{\theta}+2^{-j}\left|y-x_{j}\right|^{\theta} 2^{j+3}|| x-x_{j}|-| y-x_{j}|| \\
\leq & 2^{-j}|x-y|^{\theta}+2^{3}\left|y-x_{j}\right|^{\theta}|x-y| \\
\leq & 2^{-j}|x-y|^{\theta}+2^{3}|x-y|^{\theta}\left(\left|y-x_{j}\right|^{\theta}|x-y|^{1-\theta}\right) \\
\leq & C 2^{-j}|x-y|^{\theta}
\end{aligned}
$$

and hence

$$
|b(x)-b(y)| \leq \sum_{j=1}^{\infty}\left|b_{j}(x)-b_{j}(y)\right| \leq C|x-y|^{\theta} \sum_{j=1}^{\infty} 2^{-j} \leq C|x-y|^{\theta} .
$$

If $(-a-1) p+n>0$, then we obtain

$$
\begin{aligned}
\int_{\mathbf{B}}|\nabla u(x)|^{p} d x & \leq \sum_{j=1}^{\infty} \int_{\mathbf{B}}\left|\nabla u_{j}(x)\right|^{p} d x \\
& \leq C \sum_{j=1}^{\infty} 2^{-j p} \int_{\mathbf{B}}\left|x-x_{j}\right|^{(-a-1) p} d x \leq C \sum_{j=1}^{\infty} 2^{-j p} .
\end{aligned}
$$

Similarly, if $(-a-1+\theta) q+n>0$, then we obtain

$$
\begin{aligned}
\int_{\mathbf{B}}(b(x)|\nabla u(x)|)^{q} d x & \leq \sum_{j=1}^{\infty} \int_{\mathbf{B}}\left(b_{j}(x)\left|\nabla u_{j}(x)\right|\right)^{q} d x \\
& \leq C \sum_{j=1}^{\infty} 2^{-j q} \int_{\mathbf{B}}\left|x-x_{j}\right|^{(-a-1+\theta) q} d x \leq C \sum_{j=1}^{\infty} 2^{-j q} .
\end{aligned}
$$

Further, since $b(x) u(x) \geq 2^{-2 j}\left|x-x_{j}\right|^{-a+\theta}\left\{\varphi\left(2^{j+3}\left|x-x_{j}\right|\right)\right\}^{2}$, we see that

$$
S_{\omega}\left(b u,\left|x_{j}\right|\right)=\infty
$$

if $a>\theta+(n-1) / \omega$, which proves the claim.

We will discuss the best possibility of the exponent $\eta$ in Theorem 1.1. 
Remark 7.2. For $a>0,0<\theta \leq 1$ and $e \in \partial \mathbf{B}$, consider the function

$$
u(x)=|x-e|^{-a} \quad \text { and } \quad b(x)=|x-e|^{\theta} .
$$

Note that $|b(x)-b(y)| \leq|x-y|^{\theta}$ and $|\nabla u(x)|=a|x-e|^{-a-1}$,

$$
\int_{\mathbf{B}}|\nabla u(x)|^{p} d x<\infty
$$

when $(-a-1) p+n>0$ and

$$
\int_{\mathbf{B}}(b(x)|\nabla u(x)|)^{q} d x<\infty
$$

when $(-a-1+\theta) q+n>0$. Moreover,

$$
S_{\omega}(b u, r)=S_{\omega}\left(|\cdot-y|^{\theta-a}, r\right) \geq C(1-r)^{(\theta-a)+(n-1) / \omega}
$$

when $(\theta-a)+(n-1) / \omega<0$, or

$$
(1-r)^{\eta} S_{\omega}(b u, r) \geq C(1-r)^{(\theta-a)+(n-q) / q} .
$$

Since $(\theta-a)+(n-q) / q=(-a-1)+n / p$ can be taken sufficiently small, we see that the exponent $\eta$ in Theorem 1.1 is sharp.

Acknowledgement. We would like to express our thanks to the referee for his/her careful reading and kind comments.

\section{References}

[1] Baroni, P., M. Colombo, and G. Mingione: Regularity for general functionals with double phase. - Calc. Var. Partial Differential Equations 57:62, 2018.

[2] Baroni, P., M. Colombo, and G. Mingione: Non-autonomous functionals, borderline cases and related function classes. - St. Petersburg Math. J. 27, 2016, 347-379.

[3] Colasuonno, F., and M. Squassina: Eigenvalues for double phase variational integrals. Ann. Mat. Pura Appl. (4) 195:6, 2016, 1917-1959.

[4] Colombo, M., and G. Mingione: Regularity for double phase variational problems. - Arch. Rat. Mech. Anal. 215, 2015, 443-496.

[5] Colombo, M., and G. Mingione: Bounded minimizers of double phase variational integrals. - Arch. Rat. Mech. Anal. 218, 2015, 219-273.

[6] Gardiner, S. J.: Growth properties of $p$ th means of potentials in the unit ball. - Proc. Amer. Math. Soc. 103, 1988, 861-869.

[7] Harjulehto, P., P. Hästö, and A. Karppinen: Local higher integrability of the gradient of a quasiminimizer under generalized Orlicz growth conditions. - Nonlinear Anal. 177, 2018, $543-552$.

[8] HästÖ, P.: The maximal operator on generalized Orlicz spaces. - J. Funct. Anal. 269:12, 2015, 4038-4048; Corrigendum to "The maximal operator on generalized Orlicz spaces". - J. Funct. Anal. 271:1, 2016, 240-243.

[9] Meyers, N. G.: A theory of capacities for potentials in Lebesgue classes. - Math. Scand. 26, 1970, 255-292.

[10] Mizuta, Y.: Continuity properties of potentials and Beppo-Levi-Deny functions. - Hiroshima Math. J. 23, 1993, 79-153.

[11] Mizuta, Y.: Hyperplane means of potentials. - J. Math. Anal. Appl. 201, 1996, 226-246.

[12] Mizuta, Y.: Potential theory in Euclidean spaces. - Gakkōtosyo, Tokyo, 1996.

[13] Mizuta, Y., T. Ohno, and T. Shimomura: Growth properties for Riesz potentials of functions in weighted variable $L^{p(\cdot)}$ spaces. - Nonlinear Anal. 162, 2017, 49-75. 
[14] Mizuta, Y., T. Ohno, and T. Shimomura: Growth properties of potentials in central Morrey-Orlicz spaces on the unit ball. - Ann. Acad. Sci. Fenn. Math. 43, 2018, 1-26.

[15] Mizuta, Y., and T. Shimomura: Boundary limits of spherical means for BLD and monotone BLD functions in the unit ball. - Ann. Acad. Sci. Fenn. Math. 24, 1999, 45-60.

[16] Mizuta, Y., and T. Shimomura: Growth properties of spherical means for monotone BLD functions in the unit ball. - Ann. Acad. Sci. Fenn. Math. 25, 2000, 457-465.

[17] SERrin, J.: Local behavior of solutions of quasi-linear equations. - Acta Math. 111, 1964, $247-302$.

[18] Stoll, M.: Boundary limits of subharmonic functions in the unit disc, - Proc. Amer. Math. Soc. 93, 1985, 567-568.

[19] Yamashita, S.: Dirichlet-finite functions and harmonic functions, - Illinois J. Math. 25, 1981, 626-631.

[20] Ziemer, W. P.: Extremal length as a capacity. - Michigan Math. J. 17, 1970, 117-128.

Received 24 December 2018 • Accepted 26 March 2019 